\title{
Slowing fastest finger movements of the dominant hand with low-frequency rTMS of the hand area of the primary motor cortex
}

\author{
Jäncke, L ; et al
}

\begin{abstract}
Neuroimaging studies suggest that the primary hand motor area and the cerebellum play a pivotal role in the control of finger tapping, but their differential contribution in this task is unknown. We used therefore repetitive transcranial magnetic stimulation (rTMS) in its virtual lesion mode $(1 \mathrm{~Hz}, 10 \mathrm{~min}$, $90 \%$ of motor threshold) to study the effects of transient disruption of the right lateral cerebellum (CB), the left primary hand motor area (M1), and the right brachial plexus (PL, control site) on various finger tapping tasks (paced finger tapping task: PFT; tapping with maximum speed: TAPMAX, and tapping with convenient speed: TAPCON) in healthy right-handed subjects. RTMS of the left M1 slowed finger tapping speed of the right hand in the TAPMAX task. This effect eliminated the right hand superiority in the TAPMAX task. In addition, rTMS of the left M1 resulted in slower tapping speeds for both hands during TAPCON. There were no other effects of rTMS on tapping speed or tapping variability. Findings indicate that M1 is essential for generating fastest finger movements
\end{abstract}

DOI: https://doi.org/10.1007/s00221-003-1719-7

Posted at the Zurich Open Repository and Archive, University of Zurich

ZORA URL: https://doi.org/10.5167/uzh-156238

Journal Article

Published Version

Originally published at:

Jäncke, L; et al (2004). Slowing fastest finger movements of the dominant hand with low-frequency rTMS of the hand area of the primary motor cortex. Experimental Brain Research, 155(2):196-203.

DOI: https://doi.org/10.1007/s00221-003-1719-7 


\section{Jäncke $\cdot$ H. Steinmetz $\cdot$ S. Benilow $\cdot$ U. Ziemann \\ Slowing fastest finger movements of the dominant hand with low-frequency rTMS of the hand area of the primary motor cortex}

Received: 15 July 2003 / Accepted: 9 September 2003 / Published online: 29 November 2003

C) Springer-Verlag 2003

\begin{abstract}
Neuroimaging studies suggest that the primary hand motor area and the cerebellum play a pivotal role in the control of finger tapping, but their differential contribution in this task is unknown. We used therefore repetitive transcranial magnetic stimulation (rTMS) in its virtual lesion mode $(1 \mathrm{~Hz}, 10 \mathrm{~min}, 90 \%$ of motor threshold) to study the effects of transient disruption of the right lateral cerebellum (CB), the left primary hand motor area (M1), and the right brachial plexus (PL, control site) on various finger tapping tasks (paced finger tapping task: PFT; tapping with maximum speed: TAPMAX, and tapping with convenient speed: TAPCON) in healthy right-handed subjects. RTMS of the left M1 slowed finger tapping speed of the right hand in the TAPMAX task. This effect eliminated the right hand superiority in the TAPMAX task. In addition, rTMS of the left M1 resulted in slower tapping speeds for both hands during TAPCON. There were no other effects of rTMS on tapping speed or tapping variability. Findings indicate that M1 is essential for generating fastest finger movements.
\end{abstract}

Keywords rTMS $\cdot$ Fast finger tapping $\cdot$ Paced finger tapping $\cdot$ Asymmetry

\section{Introduction}

One of the simplest motor tasks is tapping with the index finger. Although this task is relatively undemanding it has

\footnotetext{
L. Jäncke $(\bowtie)$

Department of Neuropsychology, Institute of Psychology,

University of Zürich,

Treichlerstr. 10,

8032 Zürich, Switzerland

e-mail: 1.jaencke@psychologie.unizh.ch

Tel.: +41-1-6342192

Fax: +41-1-63444342

H. Steinmetz $\cdot$ S. Benilow $\cdot$ U. Ziemann

Clinic of Neurology, Johann Wolfgang Goethe University

Frankfurt,

Frankfurt, Germany
}

been shown that a distributed network of cortical and subcortical brain areas is involved in the control of this task (Rao et al. 1996, 1997; Jäncke et al. 1998a, 1998b; Lutz et al. 2000, 2001). Tapping has been studied using different paradigms. An often used tapping paradigm is the paced finger tapping task (PFT). In this paradigm the subjects are required to tap in synchrony with an external pacing stimulus (synchronization, S) and then continuing on their own (continuation, C) when the pacing stimulus is no longer present. This task is thought to involve the lateral cerebellum and other areas including the lateral and mesial premotor areas as essential motor control centres. Using the PFT, Ivry et al. (1988) reported that damage to the lateral cerebellum causes deficits in central processes while lesions of the medial cerebellum impair the implementation of correctly timed responses. In neurologically healthy subjects, recent functional imaging studies have shown cerebellar involvement during PFT tasks. Using repetitive transcranial magnetic stimulation (rTMS), Theoret et al. (2001) recently studied the effects of disruption of the lateral or medial cerebellum on PFT performance. These authors found greater variability on the PFT task following a 5-min train of $1 \mathrm{~Hz}$ rTMS to the medial cerebellum. Thus, there is converging evidence that the cerebellum plays an important role in motor timing processes as studied with PFT.

Another tapping paradigm is to tap with maximum speed. It has been shown that maximum tapping speed with the index finger is significantly faster for the dominant hand. That is right-handers reveal faster tapping speeds for the right than for the left hand while lefthanders show the opposite pattern. This hand skill asymmetry correlates with the size of the estimated volume of the hand motor area (Amunts et al. 1997, 2000) and is relatively stable during the course of shortterm hand skill training (Peters 1977, 1981). Investigating professional musicians, it has also been shown that this hand skill asymmetry correlates with the age at which musical training commenced (Jäncke et al. 1997) (reduced in those musicians starting very early in life with their musical training), indicating that early and intensive piano 
training might interfere with innate maturation processes that are responsible for hand skill asymmetry. However, although this hand skill marker is widely used in neuropsychological and neurological research its neurophysiological origins are currently unknown. One theory is that the larger dominant cortical hand motor area contains more motoneurons enabling a more refined and faster hand motor control (Amunts et al. 2000). Thus, it is argued that maximum tapping speed is mainly controlled by the cortical hand motor area. Several PET and fMRI experiments have shown that finger tapping rate correlates with activation within the sensorimotor cortex (including M1 and S1). Interestingly, this correlation only holds for faster tapping frequencies while for slow movements a different relationship emerged (Jäncke et al. 1998a, 1998a). In addition, single-cell recording studies in monkeys have shown that the discharge activity of M1 motoneurons maximally correlates with the force and velocity of the movement (Hepp-Reymond 1988). Since fast finger tapping requires high movement velocities one might conclude that fast finger tapping is mainly controlled by M1 motoneurons.

Taken together converging evidence points to the view that tapping involves the primary hand motor area as well as a distributed neural network involving the cerebellum and areas in the lateral and mesial premotor areas. However, although the brain areas which are involved in the control of finger tapping have been identified using modern brain imaging methods, it is not clear which particular role a specific brain area plays within this network. A possible strategy to investigate the contribution of a brain area of interest for the control of finger tapping is to block this particular brain area and to study the resulting tapping performance. This is the strategy employed in the current experiments. We used lowfrequency $(1 \mathrm{~Hz})$ rTMS to block or inhibit two particular brain areas (M1 and the lateral cerebellum) in order to examine their specific role in controlling finger tapping. As finger tapping tasks we used two different paradigms. Firstly, the PFT task was used because it is hypothesized that this task preferably requires cerebellar activation. Secondly, tapping with maximum speed was introduced because it is thought that unlike the PFT task the accomplishment of this task more strongly relies on the discharge pattern of M1. Thus, we hypothesized that inhibition of the cerebellum will affect tapping performance during the PFT task but not during tapping with maximum speed. On the other hand inhibition of M1 should affect tapping performance only for tapping with maximum speed but not for the PFT task.

\section{Materials and methods}

Subjects

Nine neurologically healthy subjects (three female) aged 2234 years participated in this experiment. All were familiar with TMS but naive to the aim of the study. All subjects were strongly right-handed (no subject with a history of familial left-handedness), as determined by a questionnaire proposed by Peters (1998). Each preference item was scored from 2 (strongly right handed) to -2 (strongly left handed). The total handedness score was calculated by dividing the sum of the scores by the maximum score achievable $(8$ items $=$ maximum score 16) resulting in a total score ranging from -1 (strongly left handed) to +1 (strongly right handed). All subjects revealed a handedness score at least larger than 0.9 , thus indicating them as strong right-handers. All subjects gave informed written consent. The experiments conformed to the Declaration of Helsinki and were approved by the local Ethics Committee.

\section{General experimental design}

The general principle of this experiment was to measure tapping performance in the context of different tasks during a "pre-TMS" session which was followed by $10 \mathrm{~min}$ of rTMS (1 Hz). Immediately after rTMS the "post-TMS" session followed during which the subjects performed the same tapping tasks as during the "pre-TMS" session. The following tapping tasks were used:

1. Tapping with maximum speed with the right index finger (TAPMAX R)

2. Tapping with maximum speed with the left index finger (TAPMAX L)

3. Tapping with convenient tapping speed with the right index finger (TAPCON R)

4. Tapping with convenient tapping speed with the left index finger (TAPCON L)

5. Synchronization tapping with the right index finger with an interstimulus interval of $500 \mathrm{~ms}$ (S R 500)

6. Continuation tapping with the right index finger with an interstimulus interval of $500 \mathrm{~ms}(\mathrm{C} \mathrm{R} 500)$

7. Synchronization tapping with the right index finger with an interstimulus interval of $1,000 \mathrm{~ms}(\mathrm{~S} R$ 1000)

8. Continuation tapping with the right index finger with an interstimulus interval of $1,000 \mathrm{~ms}(\mathrm{C} R$ 1000)

9. Synchronization tapping with the left index finger with an interstimulus interval of $500 \mathrm{~ms}(\mathrm{~S}$ L 500)

10. Continuation tapping with the left index finger with an interstimulus interval of $500 \mathrm{~ms}$ (C L 500)

11. Synchronization tapping with the left index finger with an interstimulus interval of $1,000 \mathrm{~ms}(\mathrm{~S} \mathrm{~L} \mathrm{1000)}$

12. Continuation tapping with the left index finger with an interstimulus interval of $1,000 \mathrm{~ms}\left(\mathrm{C}_{\mathrm{L}} \mathrm{L} 1000\right)$

We have used the term "convenient" to express that the subjects were free to choose a personal tapping speed. Thus, it is a fast but convenient tapping speed. Several studies have used similar tasks and found that this task is associated with different control modes as compared to tapping with maximum tapping speed (Wittmann et al. 2001). The subjects were seated comfortably in a chair. They were instructed to place both hands in front of a computer keyboard and to press the right-arrow button for the right hand conditions and the CTRL button for the left hand conditions. All conditions were fully controlled by computer software designed in the "Presentation" experimental software environment (www.neurobehavioralsystems. com). During the TAPMAX conditions a red arrow appeared on the screen pointing to the right (for right hand movements) or pointing to the left (for left hand movements). The subjects were required to tap as fast as possible with their index finger as long as the arrow was present. After $20 \mathrm{~s}$ the arrow disappeared and the subjects had to stop tapping. When a yellow arrow appeared the subjects were required to tap with convenient speed with the indicated finger. Again, after $20 \mathrm{~s}$ the arrow disappeared and the tapping task was finished. During the PFT conditions an arrow pointing to the right or the left appeared regularly with a frequency of 1 or $2 \mathrm{~Hz}$ (synchronization: S). The subjects were required to tap as precisely as possible according to the frequency of the pacing stimulus. After $30 \mathrm{~s}$ the arrow disappeared and the subjects were instructed to continue tapping with the prescribed speed (continuation: C). Thus, 
each PFT condition lasted exactly 1 min with $30 \mathrm{~s} \mathrm{~S}$ and $30 \mathrm{~s} \mathrm{C}$. The order of the 12 tapping conditions was randomized and balanced across the subjects. The entire block of tapping conditions lasted about 6 min. sites:

rTMS was applied on three consecutive days to three different

1. The hand area of the primary motor cortex (M1) on the left hemisphere

2. the lateral cerebellum $(\mathrm{CB})$ on the right, and

3. the brachial plexus (PL) on the right.

The order of rTMS sites was pseudo-randomized and counterbalanced across the subjects. PL was used as a control site in order to disentangle possible non-specific, peripheral or spinal (Gerschlager et al. 2002) from specific motor cortical or cerebellar disruptive rTMS effects.

\section{TMS}

Subjects were seated comfortably in a reclining chair. Surface EMG was recorded from the right first dorsal interosseus muscle (FDI) and the right biceps muscle with silver-silver chloride cup electrodes in a belly-tendon montage. The EMG of the biceps muscle was recorded according to safety considerations in order to detect possible spread of activation to the upper arm representation during M1 rTMS (Pascual-Leone et al. 1994). After amplification and bandpass filtering (0.1-2.5 kHz, counterpoint electromyograph; Dantec Electronics, Skovlunde, Denmark), the EMG signal was digitized (analog-to-digital rate of $5 \mathrm{kHz}, \mathrm{CED} 1401$ micro, Cambridge Electronic Devices, Cambridge, UK) and fed into a laboratory PC for on-line and off-line analysis. Focal TMS was applied to the left M1 to measure resting motor threshold (RMT) using a figure of eight-shaped stimulating coil (diameter of each wing, $70 \mathrm{~mm}$ ) connected to a "rapid transcranial magnetic stimulator" (Magstim, Whitland, Dyfed, UK). The coil was placed tangentially to the scalp with the handle pointing backward and rotated away from the midline by $45^{\circ}$. This way, the first quarter-cycle of the cosine waveform of the current induced in the brain is directed in a posterior-to-anterior direction, while the biologically more effective following half-cycle is directed in the opposite direction. The coil was moved over the hand area of the motor cortex to determine the optimal position that consistently resulted in motor evoked potentials (MEP) of maximal amplitude in the FDI. This position was marked on the scalp with a pen to ensure an identical coil placement throughout the experiment. RMT was determined to the nearest $1 \%$ of stimulator output and was defined as the minimal stimulus intensity that was sufficient to elicit MEP greater than $50 \mu \mathrm{V}$ peak-to-peak amplitude in at least five out of ten trials (Rossini et al. 1994)

After determination of RMT, a 10-min train of $1 \mathrm{~Hz}$ rTMS was delivered to one of the three stimulation sites (CB, M1, PL) at an intensity of $90 \%$ of RMT. This rTMS protocol conforms to the currently accepted safety limits (Wassermann 1998). In addition, this protocol was selected because previous experiments using the same or similar settings of rTMS duration, frequency and intensity showed a lasting inhibitory or disruptive effect of the stimulated or connected brain regions (Kosslyn et al. 1999; Gerschlager et al. 2001; Enomoto et al. 2001). For rTMS of M1 we used the same position as was used for determining RMT. For rTMS of the lateral right $\mathrm{CB}$ the centre of the coil was placed $3 \mathrm{~cm}$ rightwards to the inion and the coil was held with the handle pointing down (Werhahn et al. 1996). For stimulation of the PL, the coil was centred over the cervical spine at the level of $\mathrm{C} 7$ and the coil was rotated about $45^{\circ}$ so that the handle pointed down and to the left (Werhahn et al. 1996). In some subjects with a high RMT, it was necessary to replace the coil by another identical coil after $5 \mathrm{~min}$ of rTMS to avoid overheating.
Data analysis and statistical analysis

During all tapping conditions intertap intervals (ITI) were calculated between each pair of concomitant taps. For each condition during the "pre-TMS" and the "post-TMS" session mean ITIs and coefficients of variations (CV) were computed. The $\mathrm{CV}$ is a measure of variability correcting for differences between the means $(\mathrm{SD} \times 100 /$ mean). Thus, this variability measure can be used for between-condition and between-subjects comparisons. The ITIs and CVs of the TAPMAX and TAPCON conditions were subjected to four-way ANOVAs with repeated measurements on each factor [HAND: right vs. left; TMS: pre vs. post; SPEED: fast (TAPMAX) vs. slow (TAPCON); SITE: M1, CB, PL]. The ITIs and CVs of the PFT conditions were subjected to five-way ANOVAs with repeated measurements on each factor (HAND: right vs. left; TMS: pre vs. post; SPEED: fast $(2 \mathrm{~Hz})$ vs. slow $(1 \mathrm{~Hz})$; PACING: S vs. C; SITE: M1, CB, PL). We used this ANOVA approach as a first step to investigate whether our experimental conditions revealed the expected findings. For example, it was necessary to verify whether the subjects realized the appropriate tapping speeds and whether they correctly continued tapping during the Continuation conditions. However, because we were interested in rTMS effects we focussed our statistical analysis on the comparison between the dependent variables obtained during the pre- and post-TMS sessions. Therefore, we additionally conducted multivariate Hotellings' $t$-tests comparing ITIs and CVs obtained during pre- and post-TMS sessions. For each test (performed for each stimulation site), either ITIs or CVs were used as dependent variables. Thus, we calculated a total of 12 multivariate Hotellings' tests (three tests for the ITIs of the TAPMAX task, three tests for the ITIs of the PFT task, three tests for the CVs of the TAPMAX, and three tests for the CVs of the PFT task). Results were considered as significant at the level of $p<0.05$. In case of a significant multivariate effect post hoc $t$-tests were computed using the Bonferroni correction according to Holm (1979). None of the data violated the normality assumption necessary to conduct parametric statistical tests. However, because it is more important to demonstrate the empirical effect of our treatment rather than to calculate $p$ values, we also report effect size measures independent from sample size (Cohen 1969). As effect size measure we calculated ETA ${ }^{2}$, which is the estimated variance due to the treatment in relation to the total variance. Thus, $\mathrm{ETA}^{2}$ describes the variance which is due to the experimental effect. All statistical analyses were performed using the statistical software package SPSS PC (version 10.0).

\section{Results}

All subjects tolerated rTMS well. No overt spread to the biceps motor representation occurred in the M1 rTMS condition. The mean RMT $( \pm \mathrm{SD})$ was $46.8 \%(11.1 \%)$, $42.8 \%(5.8 \%)$ and $41.3 \%(6.0 \%)$ of maximum stimulator output in the rTMS sessions of M1, CB and PL, respectively. These values were not significantly different from each other.

\section{MEPs}

MEP amplitudes in the FDI were measured for the last 300 rTMS trials for all stimulation sites and subjects resulting in $3 \times 9$ conditions. The average MEP amplitudes obtained during M1 and PL stimulation were almost identical (mean and SD; M1:0.14 mV \pm 0.13 , PL: $0.14 \mathrm{mV} \pm 0.09$ ) while no MEP were elicited during CB stimulation $(0.003 \mathrm{mV})$. This indicates that rTMS of M1 resulted in small MEP 
which were, however, not different from the ones of the PL control site.

\section{TAPMAX}

The four-way ANOVA for the dependent variable ITI revealed a significant four-way interaction between all independent variables $\left(F_{(2,7)}=4.8, p=0.047, \mathrm{ETA}^{2}=\right.$ 0.582 ) (Table 1); thus all other main effects or interactions should be interpreted with reluctance. However, SPEED had a very strong effect on the ITIs with the slowest ITIs for the TAPCON task $\left(F_{(1,8)}=67.2, p<0.001, \mathrm{ETA}^{2}=\right.$ 0.89; mean ITIs, TAPCON: $570.6 \mathrm{~ms}$, and TAPMAX: $193.4 \mathrm{~ms})$. The interaction between TMS and HAND also revealed a significant effect $\left(F_{(1,8)}=6.4, p=0.032\right.$, ETA $^{2}=$ 0.447 ) which was qualified by slightly longer ITIs for the right hand after rTMS. There was also a SPEED $\times$ HAND interaction $\left(F_{(1,8)}=7.29, p=0.027, \mathrm{ETA}^{2}=0.477\right)$ which was qualified by a right hand advantage for the TAPMAX task while a hand advantage was absent for the TAPCON task (TAPMAX, right: $185 \mathrm{~ms}$, left: $201 \mathrm{~ms}$; TAPCON, right: $571 \mathrm{~ms}$, left: $569 \mathrm{~ms}$ ). Since the four-way interaction was significant it was necessary to examine the simple effects.

Because we were mostly interested in the influence of TMS on tapping performance we calculated multivariate Hotelling's $t$-tests comparing the ITIs obtained during the pre- and post-TMS sessions. For each anatomical site

Table 1 Means $(M)$ and standard errors of the mean $(S E)$ for the ITIs $(\mathrm{ms})$ and coefficients of variation $(\mathrm{CV}$ : $\mathrm{SD} \times 100 /$ mean ITI) obtained for the maximum and convenient tapping conditions $(\mathrm{RH}$ right hand, $L H$ left hand). The stimulation sites are abbreviated as follows: $M 1$ primary hand motor cortex, $C B$ lateral cerebellum, $P L$ brachial plexus

\begin{tabular}{llllll} 
M1 & & CB & & & \\
\cline { 1 - 2 } & ML & & \\
$M$ & SE & SE & SE
\end{tabular}

ITI (ms)

\begin{tabular}{lllllllll} 
Maximum & RH & Pre-TMS & 177.3 & 5.1 & 181.6 & 4.7 & 189.9 & 5.4 \\
& & Post-TMS & 199.1 & 6.9 & 180.1 & 4.4 & 184.2 & 2.8 \\
& LH & Pre-TMS & 199.6 & 6.4 & 194.5 & 10.4 & 207.3 & 6.9 \\
& & Post-TMS & 201.8 & 7.3 & 196.8 & 6.5 & 206.2 & 4.3 \\
Convenient & RH & Pre-TMS & 527.0 & 54.6 & 487.0 & 49.0 & 514.8 & 57.1 \\
& & Post-TMS & 630.4 & 51.7 & 642.2 & 76.7 & 629.9 & 68.9 \\
& LH & Pre-TMS & 521.5 & 52.4 & 483.7 & 46.2 & 536.5 & 58.5 \\
& & Post-TMS & 624.5 & 55.7 & 621.6 & 66.8 & 629.1 & 70.8 \\
CV (\%) & & & & & & & & \\
Maximum & RH & Pre-TMS & 21.0 & 3.4 & 18.4 & 1.8 & 15.5 & 1.2 \\
& & Post-TMS & 19.1 & 2.6 & 16.5 & 1.8 & 17.2 & 1.5 \\
& LH & Pre-TMS & 22.3 & 6.5 & 25.6 & 8.7 & 14.6 & 1.5 \\
& & Post-TMS & 16.8 & 1.7 & 17.2 & 1.7 & 21.1 & 1.7 \\
Convenient & RH & Pre-TMS & 16.9 & 5.6 & 10.1 & 1.3 & 11.3 & 1.4 \\
& & Post-TMS & 11.0 & 1.2 & 7.5 & 1.0 & 8.7 & 0.9 \\
& LH & Pre-TMS & 9.4 & 1.04 & 8.5 & 0.7 & 10.7 & 1.2 \\
& & Post-TMS & 9.0 & 1.9 & 8.0 & 0.9 & 11.6 & 2.8 \\
\hline
\end{tabular}

receiving rTMS a multivariate test was calculated. Only the Hotelling's test for rTMS of M1 revealed a significant multivariate result $\left(F_{(4,5)}=18.7, p=0.003\right.$, ETA $\left.^{2}=0.94\right)$ (Table 1). Subsequently conducted univariate $t$-tests revealed significant rTMS effects for tapping with the right hand in the TAPMAX task $\left(F_{(1,8)}=34.1, p<0.001\right.$, $\left.\mathrm{ETA}^{2}=0.81\right)$, tapping with the right hand in the TAPCON task $\left(F_{(1,8)}=9.3, p=0.016, \mathrm{ETA}^{2}=0.54\right)$, and tapping with the left hand in the TAPCON task $\left(F_{(1,8)}=12.3, p=\right.$ $\left.0.008, \mathrm{ETA}^{2}=0.61\right)$. In these three conditions, rTMS resulted in prolonged ITIs (slowed tapping speed) (Table 1, Figs. 1,2). The right hand advantage in the TAPMAX task which was present prior to rTMS disappeared after rTMS (right vs. left hand pre TMS: $t_{(8)}$ $=7.1, p<0.001$, mean difference: $22.3 \mathrm{~ms}$; right vs. left hand post TMS: $t_{(8)}=1.2, p=0.25$, mean difference: $2.7 \mathrm{~ms}$ ) (Fig. 1).

The four-way ANOVA with $\mathrm{CV}$ as the dependent variable only revealed a significant effect for SPEED $\left(F_{(1,8)}=43.6, p<0.001, \mathrm{ETA}^{2}=0.85\right)$. This effect was due to larger variability measures for the TAPMAX task (means \pm SEs for TAPCON and TAPMAX: $10.2 \pm .58 \mathrm{~ms}$ and $18.7 \pm 1.6 \mathrm{~ms}$, respectively).

\section{PFT task}

The five-way ANOVA conducted with the ITI as the dependent variable revealed a significant main effect for $\operatorname{SPEED}\left(F_{(1,8)}=1,132.03, p<0.001, \mathrm{ETA}^{2}=0.99\right)$ which was due to longer ITIs for the slow tapping task. There was no further significant main effect or interaction. It is worth noting that there was neither a significant main effect for rTMS nor a significant interaction of rTMS with one of the other factors (all $p$ values $>0.37$ ).

Subjecting the CV measures obtained during the PFT task to the five-way ANOVA revealed significant main

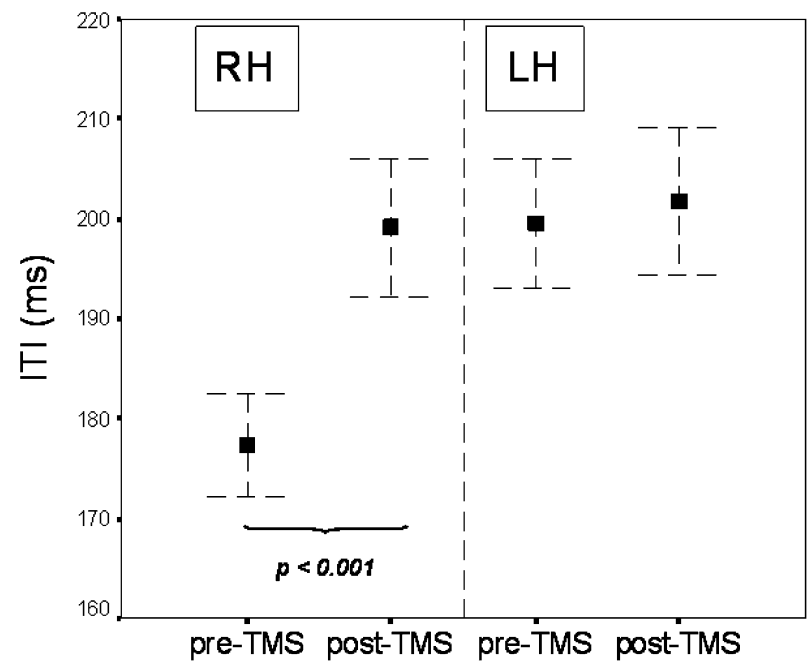

Fig. 1 Mean ITIs (in ms, filled squares) and standard errors of the mean (vertical bars through the filled squares) obtained during the TAPMAX condition for the right and left hands during the pre-TMS and post-TMS conditions (stimulation site: M1) 


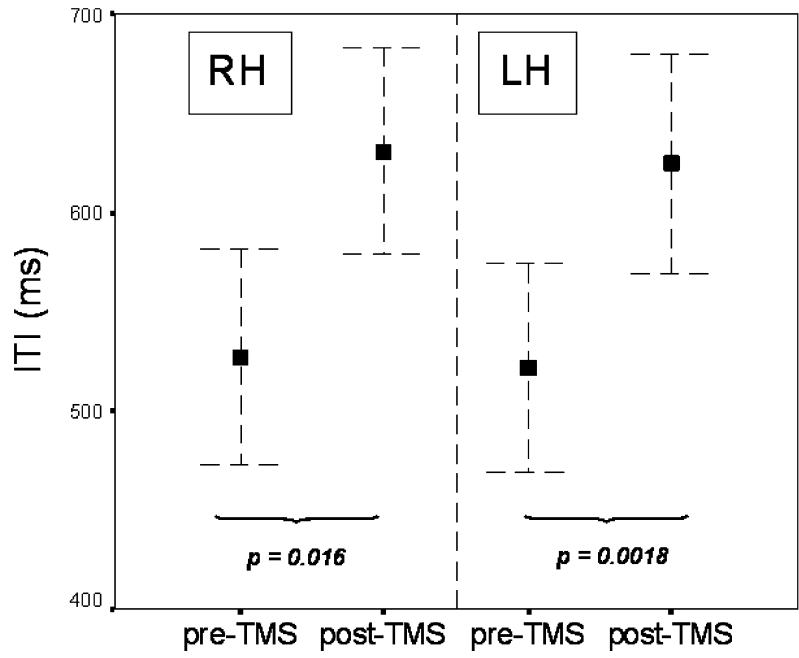

Fig. 2 Mean ITIs (in ms, filled squares) and standard errors of the mean (vertical bars through the filled squares) obtained during the TAPCON condition for the right and left hands during the pre-TMS and post-TMS conditions (stimulation site: M1)

effects for $\operatorname{SPEED}\left(F_{(1,8)}=12.8, p=0.007, \mathrm{ETA}^{2}=0.61\right)$, $\operatorname{HAND}\left(F_{(1,8)}=53.5, p<0.001, \mathrm{ETA}^{2}=0.87\right)$, and SC $\left(F_{(1,8)}=88.4, p<0.001, \mathrm{ETA}^{2}=0.92\right)$. These main effects were qualified by larger variability scores during fast tapping, for the right hand, and during synchronization. There were also two significant interaction effects: one for the two-way interaction between HAND $\times \mathrm{SC}\left(F_{(2,7)}=\right.$ 23.6, $\left.p<0.001, \mathrm{ETA}^{2}=0.75\right)$ and the other for the twoway interaction between $\operatorname{SITE} \times \operatorname{SC}\left(F_{(2,7)}=6.7, p=\right.$ $\left.0.024, \mathrm{ETA}^{2}=0.66\right)$. The two-way interaction between HAND and SC was due to a larger difference between the variability scores obtained during synchronization and continuation for the right hand than for the left hand. The interaction between SITE and SC was due to larger variability scores during synchronization during pre- and post-TMS stimulation of PL.

\section{Discussion}

We used low-frequency rTMS in its virtual lesion mode to disrupt left M1 or the right lateral cerebellum in order to examine the role of these areas in controlling different tapping movements with the right and left index finger. Consistent with our hypotheses, we found slowed finger tapping rates of the right hand during tapping with maximum speed (TAPMAX) after rTMS of the left M1. However, we also found significant slowing of tapping for both hands in the convenient speed condition (TAPCON). Tapping performance asymmetry (which is the tapping speed preponderance of the dominant right hand compared to the subdominant left hand) was absent after rTMS of the left M1 for the TAPMAX task because of the slowed tapping speed of the dominant hand. These findings are important in several respects. Firstly, this study shows that rTMS to the left M1 selectively disturbs a specific tapping task (tapping with maximum tapping speed) and does not exert an unspecific effect on other tapping tasks [paced finger tapping (PFT) remained unaffected]. Secondly, we did not find any influence of rTMS of the lateral cerebellum on either tapping task. Thus, we were unable to substantiate the notion that the cerebellum is crucial for tapping tasks, in particular for the PFT task. In the following we will discuss each point separately.

The motor tasks used in this experiment can be conceived of as evoking motor control strategies at different levels. For example, tapping with maximum speed is a basic motor task predominantly involving networks located in the primary hand motor cortex and the cerebellum. The other tapping tasks additionally rely on distributed neural networks including premotor and parietal cortical areas. Tapping with maximum speed mainly requires that neurons in the primary hand motor area generate strong activation in order to produce maximum finger movement velocities. It has been shown that movement velocity is directly related to the intensity of the discharge pattern of cortical neurons in the primary motor cortex. For instance, Humphrey et al. (1972) used multiple regression to correlate the discharge activity of several neurons in monkey M1 with the position, velocity, force, and time derivative of force in a wrist flexion and extension task with various loads. They found that linear correlations were highest for force and velocity. In addition, Ashe and Georgopolous (1994) have demonstrated that the activity of a large fraction of neurons in M1 was significantly correlated with velocity and acceleration. Given the direct relationship between velocity and frequency of movement in the TAPMAX condition, that literature provides support for our assumption that the primary hand motor cortex is mainly involved in tapping with maximum speed. If M1 controls maximum tapping speed then it comes as no surprise that transient disruption or inhibition of this network has detrimental effects on tapping performance. This effect was only present for the right hand but not the left hand, thus supporting the local specificity of the rTMS effect for the hand contralateral to the stimulated M1. RTMS effects on maximum tapping speed were reported in two previous studies. In one study, $1 \mathrm{~Hz}$ rTMS was applied to M1 or $5 \mathrm{~cm}$ anterior or posterior to M1 at a clearly supra-threshold intensity (125\% RMT) and a relatively short train was delivered (on average 204 s) (Wassermann et al. 1996). Those authors found a topographically non-specific increase in maximum tapping speed. Therefore, arousal or practice effects cannot be excluded and the meaning of this result remains unclear. The other study applied $15 \mathrm{~min}$ of $1 \mathrm{~Hz}$ rTMS at approximately $120 \%$ RMT to M1 in a sham-controlled design (Sommer et al. 2002). They found a trend towards a decrease in maximum tapping speed only in the real rTMS condition, and only for the hand contralateral to the stimulated M1 which, however, was not statistically significant. Why those authors failed to produce a significant effect is difficult to assess, but differences in the rTMS protocol and a higher mean age of the study population may have contributed. Therefore, the present data demonstrate for the first time that M1 is essentially 
involved in fastest finger tapping and that this process is topographically specific (rTMS of M1 affects contralateral hand only, no effect by rTMS of CB or PL) and task specific (PFT tasks not affected). Our finding is supported at least by two recent papers also using low-frequency rTMS of the primary motor cortex (M1). One paper revealed a lasting decrease of MEPs of the contralateral hand (Plewnia et al. 2003) while the other paper demonstrated a reduced "Bereitschaftspotential" (BP) after rTMS (Rossi et al. 2000). Thus, these papers corroborate the inhibitory influence of rTMS on M1 activation.

However, it cannot be excluded that rTMS of M1 costimulated the adjacent dorsolateral premotor area. This area has a particularly low threshold to TMS (Civardi et al. 2001; Gerschlager et al. 2001; Munchau et al. 2002). However, if such a co-stimulation occurred, it is unlikely that it was behaviourally relevant because the dorsolateral premotor area is thought to be important for externally triggered movements (Wise 1985) which, in the present study, were not affected by rTMS of M1.

In further support of an involvement of human M1 in fastest motor responses, and in excellent agreement with the present data, one recent rTMS study showed that visually cued simple reaction times were significantly slowed after a train of $20 \mathrm{~min}$ of $1 \mathrm{~Hz}$ rTMS at an intensity of $80 \%$ of active motor threshold delivered over M1, and to a weaker extent when applied to the dorsolateral premotor area (Schlaghecken et al. 2003). Similar to the present findings, this effect was specific for the contralateral hand.

Contrary to the maximum tapping speed condition, we found that, in the convenient tapping task, both hands were slower after rTMS of the left M1. The reason for this effect is not entirely clear. However, tapping with convenient tapping speed requires different and most likely more complicated control strategies as to the condition during which tapping with maximum speed is required. Several studies have shown that unimanual skilled movements with the subdominant left hand are also (at least in part) controlled by the left-sided dominant hand motor area. For example several fMRI studies (Kim et al. 1993a, 1993b; Alkadhi et al. 2002; Curt et al. 2002) showed ispilateral activation in M1 during a fingeropposition task performed with the subdominant left hand. These findings confirm earlier models explaining hand skill asymmetry. Several variants of these models propose that the dominant hand motor area either houses motor programs for both hands or that motor programs are located in the dominant motor cortex also influencing movements of the left hand (Taylor and Heilman 1980; Thut et al. 1996; Parlow and Kinsbourne 1989). Thus, we believe that tapping with convenient tapping speed with the left hand will also rely on neural networks located in the left M1. Therefore, it comes as no surprise that disturbing the dominant M1 will also affect more complicated movements performed with the subdominant hand.

Why rTMS of the left M1 did not interfere with the PFT task is not entirely clear. The PFT requires that movements are guided by external cues. This involves the ventral and dorsal parts of the lateral premotor cortex. In the monkey, the hand area of the premotor cortex is heavily reciprocally connected to the hand area of M1 (Matelli et al. 1986). While subthreshold $1 \mathrm{~Hz}$ rTMS applied to the premotor cortex can alter M1 excitability (Gerschlager et al. 2001; Munchau et al. 2002) little is known to what extent rTMS of M1 has effects on neural activity in the premotor cortex. One recent study showed that subthreshold $1 \mathrm{~Hz}$ rTMS of M1 resulted in a reduced sensitivity of the stimulated cortex to input from distant areas, in particular the premotor cortex (Lee et al. 2003). Another study demonstrated that EEG-EEG coherence in the alpha-band increased between M1 and premotor cortex after subthreshold $1 \mathrm{~Hz}$ rTMS of M1, indicating enhanced inter-regional inhibition (Strens et al. 2002). However, it is likely that functional deficits that may arise from these alterations in connectivity between M1 and premotor cortex are compensated for by acute re-mapping of connectivity between the premotor areas and parts of M1 (Lee et al. 2003). Such a compensatory re-mapping would explain why PFT performance remained unaltered after rTMS of M1 in the present experiments.

Another important finding of our study is that rTMS of the lateral cerebellum did not influence tapping speed in any condition. Thus, we were unable to support the notion that the cerebellum plays a crucial role in controlling tapping movements. According to lesion and neuroimaging studies we had anticipated that the lateral cerebellum might be involved at least in the control of the PFT task. That we were not able to disrupt the PFT task by stimulating the lateral cerebellum might have several reasons. First, it might be that the lateral cerebellum is indeed not essentially involved in controlling the performance during the PFT task. This idea is supported to some extent by other authors who argue that especially the cerebellar hemispheres of the cerebellum are working like parallel computers subserving and supporting the control demands of other motor control centres. Thus, the cerebellum is not essential but only supportive in controlling timed motor responses. Disrupting the cerebellum would not disrupt the core control process but rather a subsidiary part of motor control. Secondly, it might also be that not the lateral but rather the medial cerebellum is stronger involved in controlling timed motor responses. For example, Theoret et al. (2001) recently found increased intertap variability after rTMS of the mesial cerebellum but not the lateral cerebellum. A third possible reason for the lack of effect after stimulating the lateral cerebellum might be that we have used too low rTMS intensities. This may be suggested from previous investigations which showed, for instance, that activation of the Purkinje cell projection to the deep cerebellar nuclei required relatively high stimulus intensities (Ugawa et al. 1995). Thus, future experiments should try to optimize stimulation parameters in order to disturb cerebellar processes more efficiently. In addition, it is also necessary to stimulate other motor areas which have been shown to be involved in motor timing. Interesting candidates for inhibition are the mesial and lateral premotor areas. 


\section{References}

Alkadhi H, Crelier GR, Boendermaker SH, Hepp-Reymond MC, Kollias SS (2002) Somatotopy in the ipsilateral primary motor cortex. Neuroreport 13:2065-2070

Amunts K, Schlaug G, Jäncke L, Steinmetz H, Schleicher A, Zilles K (1997) Hand skills covary with the size of motor cortex: a macrostructural adaptation. Hum Brain Mapp 5:206-215

Amunts K, Jäncke L, Mohlberg H, Steinmetz H, Zilles K (2000) Interhemispheric asymmetry of the human motor cortex related to handedness and gender. Neuropsychologia 38:304-312

Ashe J, Georgopoulos AP (1994) Movement parameters and neural activity in motor cortex and area 5. Cereb Cortex 4:590-600

Civardi C, Cantello R, Asselman P, Rothwell JC (2001) Transcranial magnetic stimulation can be used to test connections to primary motor areas from frontal and medial cortex in humans. Neuroimage 14:1444-1453

Cohen J (1969) Statistical power analysis for the behavioral sciences. Academic Press, New York

Curt A, Alkadhi H, Crelier GR, Boendermaker SH, Hepp-Reymond MC, Kollias SS (2002) Changes of non-affected upper limb cortical representation in paraplegic patients as assessed by fMRI. Brain 125:2567-2578

Enomoto H, Ugawa Y, Hanajima R, Yuasa K, Mochizuki H, Terao Y, Shiio Y, Furubayashi T, Iwata NK, Kanazawa I (2001) Decreased sensory cortical excitability after $1 \mathrm{~Hz}$ rTMS over the ipsilateral primary motor cortex. Clin Neurophysiol 112:2154-2158

Gerschlager W, Siebner HR, Rothwell JC (2001) Decreased corticospinal excitability after subthreshold $1 \mathrm{~Hz}$ rTMS over lateral premotor cortex. Neurology 57:449-455

Gerschlager W, Christensen LO, Bestmann S, Rothwell JC (2002) rTMS over the cerebellum can increase corticospinal excitability through a spinal mechanism involving activation of peripheral nerve fibres. Clin Neurophysiol 113:1435-1440

Hepp-Reymond MC (1988) Functional organization of motor cortex and its participation in voluntary movements. In: Seklis HD, Erwin J (eds) Comparative primate biology. Liss, New York, pp $501-624$

Holm S (1979) A simple sequentially rejective multiple test procedure. Scand J Stat 6:65-70

Humphrey DR (1972) Relating motor cortex spike trains to measures of motor performance. Brain Res 40:7-18

Ivry RB, Keele SW, Diener HC (1988) Dissociation of the lateral and medial cerebellum in movement timing and movement execution. Exp Brain Res 73:167-180

Jäncke L, Schlaug G, Steinmetz H (1997) Hand skill asymmetry in professional musicians. Brain Cogn 34:424-432

Jäncke L, Peters M, Schlaug G, Posse S, Steinmetz H, MullerGartner H (1998a) Differential magnetic resonance signal change in human sensorimotor cortex to finger movements of different rate of the dominant and subdominant hand. Brain Res Cogn Brain Res 6:279-284

Jäncke L, Specht K, Mirzazade S, Loose R, Himmelbach M, Lutz K, Shah NJ (1998b) A parametric analysis of the 'rate effect' in the sensorimotor cortex: a functional magnetic resonance imaging analysis in human subjects. Neurosci Lett 252:37-40

Kim SG, Ashe J, Georgopoulos AP, Merkle H, Ellermann JM, Menon RS, Ogawa S, Ugurbil K (1993a) Functional imaging of human motor cortex at high magnetic field. J Neurophysiol 69:297-302

Kim SG, Ashe J, Hendrich K, Ellermann JM, Merkle H, Ugurbil K, Georgopoulos AP (1993b) Functional magnetic resonance imaging of motor cortex: hemispheric asymmetry and handedness. Science 261:615-617

Kosslyn SM, Pascual-Leone A, Felician O, Camposano S, Keenan JP, Thompson WL, Ganis G, Sukel KE, Alpert NM (1999) The role of area 17 in visual imagery: convergent evidence from PET and rTMS. Science 284:167-170
Lee L, Siebner HR, Rowe JB, Rizzo V, Rothwell JC, Frackowiak RS, Friston KJ (2003) Acute remapping within the motor system induced by low-frequency repetitive transcranial magnetic stimulation. J Neurosci 23:5308-5318

Lutz K, Specht K, Shah NJ, Jäncke L (2000) Tapping movements according to regular and irregular visual timing signals investigated with fMRI. Neuroreport 11:1301-1306

Lutz K, Weidner R, Shah NJ, Jäncke L (2001) The transfer of a timing pattern to the untrained human hand investigated with functional magnetic resonance imaging. Neurosci Lett 301:4548

Matelli M, Camarda R, Glickstein M, Rizzolatti G (1986) Afferent and efferent projections of the inferior area 6 in the macaque monkey. J Comp Neurol 251:281-298

Munchau A, Bloem BR, Irlbacher K, Trimble MR, Rothwell JC (2002) Functional connectivity of human premotor and motor cortex explored with repetitive transcranial magnetic stimulation. J Neurosci 22:554-561

Parlow SE, Kinsbourne M (1989) Asymmetrical transfer of training between hands: implications for interhemispheric communication in normal brain. Brain Cogn 11:98-113

Pascual-Leone A, Valls-Sole J, Wassermann EM, Hallett M (1994) Responses to rapid-rate transcranial magnetic stimulation of the human motor cortex. Brain 117:847-858

Peters M (1977) Simultaneous performance of two motor activities: the factor of timing. Neuropsychologia 15:461-465

Peters M (1981) Handedness: coordination of within- and betweenhand alternating movements. Am J Psychol 94:633-643

Peters M (1998) Description and validation of a flexible and broadly usable hand preference questionnaire. Laterality 3:77-96

Plewnia C, Lotze M, Gerloff C (2003) Disinhibition of the contralateral motor cortex by low-frequency rTMS. Neuroreport 14:609-612

Rao SM, Bandettini PA, Binder JR, Bobholz JA, Hammeke TA, Stein EA, Hyde JS (1996) Relationship between finger movement rate and functional magnetic resonance signal change in human primary motor cortex. J Cereb Blood Flow Metab 16:1250-1254

Rao SM, Harrington DL, Haaland KY, Bobholz JA, Cox RW, Binder JR (1997) Distributed neural systems underlying the timing of movements. J Neurosci 17:5528-5535

Rossi S, Pasqualetti P, Rossini PM, Feige B, Ulivelli M, Glocker FX, Battistini N, Lucking CH, Kristeva-Feige R (2000) Effects of repetitive transcranial magnetic stimulation on movementrelated cortical activity in humans. Cereb Cortex 10:802-808

Rossini PM, Barker AT, Berardelli A, Caramia MD, Caruso G, Cracco RQ, Dimitrijevic MR, Hallett M, Katayama Y, Lucking $\mathrm{CH}$ (1994) Non-invasive electrical and magnetic stimulation of the brain, spinal cord and roots: basic principles and procedures for routine clinical application. Report of an IFCN committee. Electroencephalogr Clin Neurophysiol 91:79-92

Schlaghecken F, Munchau A, Bloem BR, Rothwell J, Eimer M (2003) Slow frequency repetitive transcranial magnetic stimulation affects reaction times, but not priming effects, in a masked prime task. Clin Neurophysiol 114:1272-1277

Sommer M, Kamm T, Tergau F, Ulm G, Paulus W (2002) Repetitive paired-pulse transcranial magnetic stimulation affects corticospinal excitability and finger tapping in Parkinson's disease. Clin Neurophysiol 113:944-950

Strens LH, Oliviero A, Bloem BR, Gerschlager W, Rothwell JC, Brown P (2002) The effects of subthreshold $1 \mathrm{~Hz}$ repetitive TMS on cortico-cortical and interhemispheric coherence. Clin Neurophysiol 113:1279-1285

Taylor HG, Heilman KM (1980) Left-hemisphere motor dominance in righthanders. Cortex 16:587-603

Theoret H, Haque J, Pascual-Leone A (2001) Increased variability of paced finger tapping accuracy following repetitive magnetic stimulation of the cerebellum in humans. Neurosci Lett 306:2932

Thut G, Cook ND, Regard M, Leenders KL, Halsband U, Landis T (1996) Intermanual transfer of proximal and distal motor engrams in humans. Exp Brain Res 108:321-327 
Ugawa Y, Uesaka Y, Terao Y, Hanajima R, Kanazawa I (1995) Magnetic stimulation over the cerebellum in humans. Ann Neurol 37:703-713

Wassermann EM (1998) Risk and safety of repetitive transcranial magnetic stimulation: report and suggested guidelines from the International Workshop on the Safety of Repetitive Transcranial Magnetic Stimulation, June 5-7, 1996. Electroencephalogr Clin Neurophysiol 108:1-16

Wassermann EM, Grafman J, Berry C, Hollnagel C, Wild K, Clark K, Hallett M (1996) Use and safety of a new repetitive transcranial magnetic stimulator. Electroencephalogr Clin Neurophysiol 101:412-417
Werhahn KJ, Taylor J, Ridding M, Meyer BU, Rothwell JC (1996) Effect of transcranial magnetic stimulation over the cerebellum on the excitability of human motor cortex. Electroencephalogr Clin Neurophysiol 101:58-66

Wise SP (1985) The primate premotor cortex: past, present, and preparatory. Annu Rev Neurosci 8:1-19

Wittmann M, Von Steinbuchel N, Szelag E (2001) Hemispheric specialisation for self-paced motor sequences. Brain Res Cogn Brain Res 10:341-344 